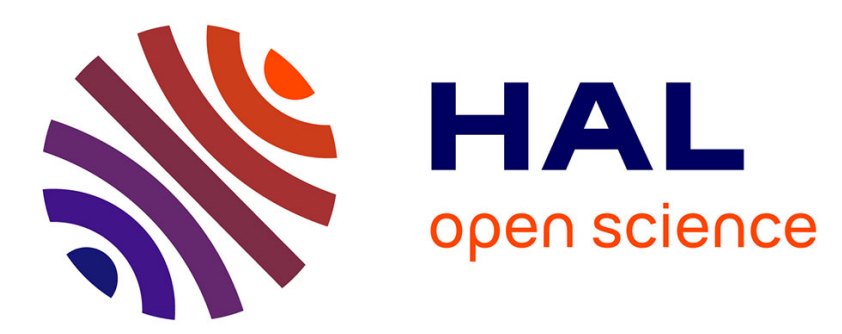

\title{
Les révolutions du salariat : du paiement à la pièce à la socialisation du salaire horaire
}

Claude Didry

\section{To cite this version:}

Claude Didry. Les révolutions du salariat : du paiement à la pièce à la socialisation du salaire horaire. Regards croisés sur l'économie, 2021, nº 27 (2), pp.32-39. 10.3917/rce.027.0032 . halshs-03506501

\section{HAL Id: halshs-03506501 https://shs.hal.science/halshs-03506501}

Submitted on 2 Jan 2022

HAL is a multi-disciplinary open access archive for the deposit and dissemination of scientific research documents, whether they are published or not. The documents may come from teaching and research institutions in France or abroad, or from public or private research centers.
L'archive ouverte pluridisciplinaire HAL, est destinée au dépôt et à la diffusion de documents scientifiques de niveau recherche, publiés ou non, émanant des établissements d'enseignement et de recherche français ou étrangers, des laboratoires publics ou privés. 


\title{
Les révolutions du salariat : du paiement à la pièce à la socialisation du salaire horaire
}

\section{Compensating work: from piecework to hourly wages}

\author{
CLAUDE DIDRY \\ Sociologue, directeur de recherche au CNRS \\ Membre du Centre Maurice Halbwachs
}

\section{Résumé}

Comment le salariat est-il devenu la forme majoritaire de rémunération du travail ? Du louage d’ouvrage formalisé dans le Code civil en 1804 au salaire encadré par un contrat de travail, la succession des formes dominantes de rémunération des activités productives révèle une évolution dans la signification même du travail.

\section{Abstract}

How did salaries become the main form of compensation? From piece wages to salaries specified by employment contracts, a historical account of successive means of compensating work reveals the shifting significance of work itself.

Dans l'Algérie en guerre, le « vrai travail » - aux yeux des paysans déplacés par l'armée française - se présente sous les traits du salariat, c'est-à-dire sous la forme (aujourd'hui familière) de la rémunération régulière que procure une activité professionnelle (Bourdieu, 1977). Dans la France de la Révolution, c'est au « vrai louage » qu'aspirent les ouvriers, c'est-à-dire au louage d'ouvrage ${ }^{1}$ à « prix fait ». Pour Karl Marx, il s'agit dans les deux cas d'un salaire : salaire au temps dans le salariat rêvé par les uns, salaire à la pièce dans le « vrai louage » revendiqué par les autres. Selon lui, l'exploitation ${ }^{2}$ est au rendez-vous

${ }^{1}$ Le louage d'ouvrage sera défini par le Code civil comme « un contrat par lequel l'une des parties s'engage à faire quelque chose pour l'autre, moyennant un prix convenu entre elles » (art. 1710).

${ }^{2}$ Voir encadré p.-, Ndlr. 
dans les deux cas, avec, à la clé, le profit des capitalistes. Mais les choses sont moins simples qu'il n'y paraît, car le salaire à la pièce s'accompagne d'une multiplication des intermédiaires, les ouvriers en embauchant d'autres pour réaliser leurs ouvrages. De sorte que "l'exploitation des travailleurs par le capital se réalise ici au moyen de l'exploitation du travailleur par le travailleur » (Marx, 1993 [1867], p. 620). Le constat est troublant et conduit à se demander si, dans ce capitalisme d' « intermédiaires » entretenu par le salaire à la pièce, il est possible de parler de salariat, malgré l'impossibilité d'identifier un salarié et un employeur. Les formes dominantes prises par la rémunération des activités productives ne révèlent-elles pas finalement des univers différents, dans lesquels se joue jusqu'à la signification du travail lui-même?

Pour répondre à cette question, une exploration historique nous conduira de la rémunération à la pièce associée à une production marchande qui s'inscrit dans la sphère familiale, au salaire prévu en contrepartie d'un travail par un contrat liant un travailleur à un employeur.

\section{Acte 1. La Révolution française et la revendication d'un « vrai}

\section{louage »}

En août 1789, les châteaux brûlent dans les campagnes et l'Assemblée nationale constituante décrète la destruction du régime féodal. Finalement, l'accès à la propriété de la terre stabilise une société majoritairement rurale et « le résultat définitif fut que la France, au contraire de l'Angleterre, devint le pays de la petite et moyenne ferme " (Weber, 1991 [1923], p. 126). Toutefois, cela ne fait que conforter une ruralité « industrieuse » encouragée par le colbertisme (Minard, 1998). Au cours du siècle suivant, le tissage de la soie continue à progresser dans les campagnes lyonnaises pour contourner l'agitation d'un artisanat urbain particulièrement revendicatif (Noiriel, 1986). La production marchande s'inscrit alors dans le temps libre que laissent les occupations agricoles, et procure des revenus qui complètent une production de subsistance.

"Nous ne sommes pas des esclaves!", "Nous ne sommes pas des domestiques!", la Révolution est soutenue par une agitation ouvrière urbaine portant une revendication de « vrai louage » contre une discipline corporative pénalisant la rupture de son engagement 
par l'ouvrier à travers le délit de désertion (Cottereau, 2002, p. 1534) ${ }^{3}$. En 1804, le louage d'ouvrage défini par les articles 1779 à 1799 du Code civil entérine une forme $d^{\prime}$ indépendance ouvrière, en disposant que les « ouvriers qui font directement des marchés à prix fait [...] sont entrepreneurs en la partie qu'ils traitent » (art. 1799). L'article 1780 sur "le louage des ouvriers et des domestiques », en prohibant les engagements perpétuels, ouvre la voie à la grève comme exercice collectif de la rupture du louage par des ouvriers revendiquant la fixation collective du prix des ouvrages. Enfin, ce contrat convient bien à une production marchande dominée par l'activité à domicile, où la réalisation de l'ouvrage s'inscrit dans les occupations familiales, les ouvriers associant les membres de la famille à la tâche.

\section{Acte 2. 1848, l'impossible prohibition du marchandage}

Il arrive qu'un ouvrier en engage d'autres pour réaliser son ouvrage, formant des chaînes de louage et instaurant un capitalisme d' «intermédiaires ". On parle de " marchandage ", un phénomène qui mine l'unité ouvrière (Dewerpe, 1989). Le décret du 2 mars 1848, dans une République qui proclame la Fraternité, entend abolir le marchandage comme exploitation de l'ouvrier par l'ouvrier. Le remède à cette exploitation est l'« association " ${ }^{4}$, ou plus tard, la " coopération » qu'encouragera la Deuxième République, sous l'impulsion de députés tels que Proudhon ou Corbon dans une perspective d'émancipation sociale (Hayat, 2014). Mais, ce décret, dont la légalité demeure contestée sous le Second Empire, n'empêche pas une diffusion du marchandage jusque dans les grandes industries naissantes. Le tableau que dresse Marx au sujet du « salaire à la pièce » dans Le Capital est ici éclairant :

« D’une part, le salaire aux pièces facilite l'intrusion de parasites entre le capitaliste et l'ouvrier salarié, le sous-affermage du travail (subletting of

labour). [...] Ce système porte en Angleterre le nom caractéristique de sweating-system. D'autre part, le salaire à la pièce permet au capitaliste de

\footnotetext{
${ }^{3}$. Contrairement à une croyance répandue, le régime des corporations repose donc sur la répression des grèves, ou « cabales ".

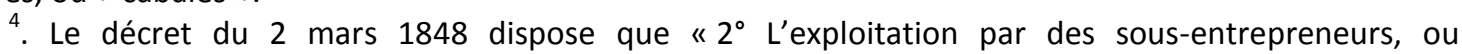
marchandage, est aboli » et préserve l'association de plusieurs ouvriers se partageant les bénéfices d'un ouvrage. Plus concrètement, le décret visait à prévenir les abus du marchandage en combinant à son abolition une réduction du temps de travail.
} 
conclure avec l'ouvrier principal [...] un contrat à tant la pièce, à un prix pour lequel l'ouvrier principal se charge lui-même de recruter et de payer ses auxiliaires. » (Marx, 1993 [1867], p. 620)

Ce capitalisme d' « intermédiaires » dépasse une production marchande artisanale. Le roman Germinal (Zola, 1885) montre le marchandage au cœur de l'activité minière, sous la forme d'une rémunération à la tonne de charbon extraite par l'équipe du Maheu. II est courant que les ouvriers spécialistes de la métallurgie recrutent des auxiliaires payés sur la base d'une rémunération collective à la pièce, jusque dans les grandes installations sidérurgiques. Mais, en 1901, le grand arrêt de la Cour de cassation sur le marchandage (Pélissier et al., 2008) ébranle sérieusement la figure du louage d'ouvrage, établissant enfin la légalité du décret de 1848.

\section{Acte 3. 28 décembre 1910, la naissance du Code du travail}

En parlant d'un code du travail dans la proposition qu'il dépose à la Chambre en 1898, Arthur Groussier sort de la grammaire de l'ouvrage. Le champ sur lequel s'exerce la codification suppose une définition du travail que la proposition Groussier fixe en ces termes :

« Article 4. Le travail s'entend de toute activité manuelle ou intellectuelle, par laquelle une personne concourt à la production, l'extraction, le façonnage, la transformation, le transport, l'emmagasinement ou la vente de matières ou produits. »(Groussier, 1898, p. 1424).

De 1901 à 1904, la commission de « codification des lois ouvrières et de prévoyance sociale ", aboutit aussi à un " code du travail » qui fournit la matrice du code adopté à partir de la loi du 28 décembre 1910. Elle introduit le terme " contrat de travail » dans le premier livre. Le contrat de travail - sans être véritablement fixé par le législateur - est alors défini par les juristes comme "le contrat par lequel une personne s'engage à travailler pour une autre qui s'oblige à lui payer un salaire calculé, soit à raison de la durée de son travail, soit à proportion de la quantité ou de la qualité de l'ouvrage accompli, soit d'après toute autre base arrêtée entre l'employeur et l'employé » (Société d'Études Législatives 1906, p. 507508 , souligné par l'auteur). Le contrat de travail se caractérise par sa généralité, en trouvant à s'appliquer tant pour l'ouvrier que pour l'ingénieur. Le marchandage se résout ainsi en un 
contrat d'équipe, dont le chef et tous les membres se trouvent individuellement liés à un même employeur.

Ces réflexions, que sous-tend l'adoption d'un Code du travail en France, introduisent une représentation nouvelle du travail ${ }^{5}$ comme participation d'un travailleur individuel à une production marchande au sein de ce que l'on commence à nommer une entreprise. On peut penser que l'essor inédit de l'industrie dans la première moitié $d u x^{e}$ siècle en France $y a$ trouvé un appui substantiel ${ }^{6}$, concomitamment à une reconfiguration du syndicalisme autour du travail et de l'industrie (ou branche), en abandonnant le métier. Le contrat de travail permet également de rattacher au salariat nombre de travailleurs à domicile, en identifiant l'employeur au donneur d'ordres principal.

Plus tard, la création d'assurances sociales dans les années 1930, unifiées dans une sécurité sociale à la Libération, s'avère décisive pour établir le champ du contrat de travail comme base d'une " socialisation du salaire " par la cotisation sociale et la garantie d'un revenu de remplacement (Friot, 2012).

\section{Conclusion}

Si les révolutions sociales et politiques qui ont traversé la France du $\mathrm{XIX}^{\mathrm{e}}$ siècle ont contribué à inscrire les activités productives dans un régime de liberté contractuelle, le Code du travail élaboré au début $d u x x^{\mathrm{e}}$ siècle a structuré la catégorie du salariat qui, dans une population active dominée par l'emploi salarié stable, a acquis aujourd'hui la force de l'évidence. En ce sens, le contrat de travail et son corollaire, le salaire, tels qu'ils ressortent de l'œuvre législative de la Troisième République, constituent une de ces « révolutions symboliques » imposant " dès lors qu'elle réussit, de nouvelles structures cognitives qui, du fait qu'elles se généralisent, qu'elles se diffusent, qu'elles habitent l'ensemble des sujets percevant d'un univers social, deviennent imperceptibles » (Bourdieu, 2013).

\section{Bibliographie}

BOURDIEU P. (1977), Algérie 60. Structures économiques et structures temporelles, Les Éditions de Minuit, Paris.

\footnotetext{
${ }^{5}$ Nous y voyons un moment déterminant pour une institution du travail (Didry, 2016).

${ }^{6}$ Métallurgie, électricité et automobile se développent, et « De 1906 à 1931, l'effectif des salariés de l'industrie a cru fortement, de 3679000 à 5442000 »(Woronoff 1998, p. 410).
} 
Bourdieu P. (2013), Manet, une révolution symbolique. Cours au Collège de France (1998-2000), Seuil, Paris.

Cottereau A. (2002), « Droit et bon droit. Un droit des ouvriers instauré, puis évincé par le droit du travail (France, XIX ${ }^{\mathrm{e}}$ siècle) ", Annales. Histoire, Sciences Sociales, 57-6, 2002, p. 1521-1561.

Dewerpe A. (1989), Le monde du travail en France, 1800-1950, Armand Colin, Paris.

DIDRY C. (2016), L'institution du travail, La Dispute, Paris.

Friot B. (2012), L'Enjeu du Salaire, La Dispute, Paris.

Groussier A. (1898), "Proposition de loi sur le code du travail ", Journal officiel, « Documents parlementaires », annexe 33, 13 juin 1898, p. 1420-1457.

HayAT S. (2014), 1848, Quand la République était révolutionnaire. Citoyenneté et représentation, Seuil, Paris.

MARX K. (1993 [1867]), Le Capital. Critique de l'économie politique, Livre 1, PUF, Paris.

MinARD P. (1998), La fortune du colbertisme. État et industrie dans la France des Lumières, Fayard, Paris.

NoIRIEL G. (1986), Les ouvriers dans la société française, $x I x^{e}-x x^{e}$ siècles, Seuil, Paris.

Pelissier J., Lyon-Caen G., Jeammaud A. et Dockes E. (2008), Les Grands arrêts du droit du travail, Dalloz, Paris.

SOCIETE d’ÉTUdes LegISLATIVES (1906), « Le projet de loi du Gouvernement et le projet de la Société d'études législatives ", Bulletin de la Société d'études législatives, p. 507-529.

WeBER M. (1991 [1923]), Histoire économique. Esquisse d'une histoire universelle de l'économie et de la société, traduction C. Bouchindhomme, Gallimard, Paris.

Woronoff D. (1994, Histoire de l'industrie en France. Du XVI siècle à nos jours, Seuil, Paris.

ZoLA E. (1999 [1885]), Germinal, Gallimard, Paris. 